the thunderbolt" to the summer solstice. The references to this god in the Rig-Veda, when divested of all allegory, suggest that he is the god of the summer solstice, while the clouds as represented by a demon are unwilling to yield up their watery store until assailed by the thunderbolt hurled by the god.

The monsoons which bring the rains usually burst about June 22 and there is usually a drought which lasts for about a month before the monsoon comes. The demon Susna (drought) is killed by Indra. The fight with Vrtra or Ahi, the cloud demon, is thus an annual affair which takes place when the sun enters the summer solstice, Indra withdrawing his raingiving (or annual) bow with the coming of autumn.

When did Indra become the slayer of Vrtra? The answer given by the Rig-Veda is when Indra by the rising of Maghas became Maghavan. Maghas to us must be the constellation Maghās consisting of $\alpha, \eta, \gamma, \zeta, \mu$ and $\varsigma$ Leonis, at the heliacal rising of which the sun reached the summer solstice at the latitude of Kuruksetra (lat. $30^{\circ} \mathrm{N}$.). This it is shown must have happened in 4170 B.c.

\title{
APPLICATIONS OF PHOTOGRAPHY TO ENGINEERING
}

T a paper delivered to the Junior Institution of 1 Engineers on November 4 and published in the journal of the Institution for December, Mr. A. H. Styring gives an account of several practical applications of photography to engineering. He lays special stress on infra-red photography, photography by polarized light, X-ray photography and high-speed photography.

Infra-red sensitive materials have proved of the greatest value in spectroscopy and astronomy. Photographs of the solar spectrum have revealed lines as far as 13,500 A. wave-length. Distance appears hazy on non-colour sensitive materials because the suspended particles of water vapour in the atmosphere, which are transparent and therefore affect vision only slightly, act as a thick medium for deep violet and ultra-violet waves, scattering them, and producing an effect similar to that which would be seen if we were to try to look through a sheet of finely ground glass.

When photographing at great distances, the best results are obtained with infra-red sensitive materials and filters. Infra-red waves are less easily scattered by water and dust particles in the intervening atmosphere than are the shorter rays in the normal visibility range. The power of haze penetration is important and has an engineering application. Research is in progress for the adaptation of infrared sensitive materials for navigational purposes. Good work has been done up to six miles, and distances up to twenty miles have been claimed. Special infra-red cameras have been fitted to transAtlantic liners in which infra-red film is exposed at 20 -second intervals, each picture being developed and fixed rapidly for viewing in roughly one minute. In the same way application has also been made for military purposes, permitting vision of objects invisible to the eye. Other applications depend mainly on the fact that many dyes and pigments, which absorb strongly in the visible region, either transmit or reflect freely in the near infra-red region. In consequence it is possible to reveal by infra-red photography many structures invisible to the eye, and to differentiate between materials which to the eye appear similar. This has a useful application in photomicrography. Another promising field lies in the direction of research in the realm of elevated temperatures. Heat rays are recorded by infra-red materials.

Polarized light photography is based on the fact that light reflected from a non-metallic surface at an angle of $32-37^{\circ}$ is strongly polarized. At other angles except $90^{\circ}$ it is to variable extents. For this reason the reflection from natural things, separately and combined, has much of the light polarized. The unaided eye fails to detect this, but many objects viewed by a polarizing screen assume a new and strange beauty. The use of polarized light in photography is made possible by the way in which all natural substances reflect polarized light. When a ray of light falls on a sheet of paper, for example, the light that is reflected is composed of two parts, which are called 'specular' and 'diffused' components. The specular component produces 'gloss' and enables us to see more or less distinctly an image of the source of light. If we look at our object through a polarizing screen, we find that we can orient the screen so that practically all the specular reflection is stopped and we see the object by diffusely reflected light. Polarizing screens have recently been made from a quinineiodine compound called 'Herapathite', a thin layer of the substance being cemented between two sheets of plane glass. The use of such a screen permits photographs to be taken obliquely through water or glass.

The principal application of X-rays to. industry is the examination of articles during manufacture. Increased penetration is constantly being achieved and the definition of radiographs has been greatly improved. It does not necessitate expensive castings or forgings being either sawn asunder or otherwise destroyed or mutilated. With a little experience, defects such as gas cavities, sand and slag inclusions, metal segregation, etc., can be easily detected. The atomic weight of the substance examined has a marked effect on the depth of penetration of the rays. Apart from this fact, penetration depends on the voltage applied to the tube. For example, 40,000 volts will penetrate 4 in. of aluminium, but it requires 250,000 volts to penetrate $4 \mathrm{in}$. of steel. The gamma radiations of radioactive substances have latterly been employed for the same purposes as $\mathrm{X}$-rays. Owing to their shorter wave-length the gamma rays have a much greater penetrative power.

The author points out that with high-speed photography, the ordinary type of motion camera which has an intermittent film motion and shutter can only be speeded up to ten times its normal speed before rupture takes place. Cameras operating at higher speeds have continuously moving films. In the mechanism of the high-speed camera perfected by the Western Electric Co. and Kodak Ltd. there is incorporated a clock, and this records the exact time when each event in a cycle takes place. 